\title{
Miguel A. Martínez, Squatters in The Capitalist City. Housing, Justice, and Urban Politics, New York and London: Routledge/ Taylor \& Francis Group, 2020
}

\author{
Robert González García \\ Universidad Autónoma del Estado de Hidalgo, México \\ rgonza67@gmail.com
}

El estudio de los okupas o del movimiento okupa ha ido ganando terreno en el campo de la Sociología y de las ciencias sociales en las últimas décadas. Y gracias en gran medida al autor de esta obra, Miguel A. Martínez, quien ha dedicado una gran parte de su carrera académica al estudio de este particular sujeto político de los movimientos sociales urbanos. De hecho, este libro contiene de forma sintética las principales aportaciones de este autor en los últimos 20 años de una brillante e itinerante trayectoria que le ha llevado a universidades nacionales como la Universidad de A Coruña, la Universidad de la Rioja, la Universidad Complutense de Madrid, e internacionales, como la Universidade do Porto, la City University of Hong Kong y la Universidad de Uppsala en Suecia, donde tiene actualmente su plaza de catedrático de sociología en el Institute for Housing and Urban Research.

Como nos señala en la introducción de la obra, desde que en 1989 entró por primera vez en Minuesa, un Centro Social Okupado (CSO) en Madrid, su curiosidad sociológica y vital por la okupación le llevó a involucrarse como académico y activista, animando investigaciones, seminarios y redes de investigación activista, tanto a nivel local, como internacional, especialmente en Europa, pero también en Asia y América Latina. La red de académicos y activistas okupas SqEK (Squatting Everywhere Kollective) ha sido la cuna de una colaboración muy fructífera entre el activismo y la investigación académica que ha reforzado a ambos, legitimando y consolidando la okupación de inmuebles abandonados para darles un uso social o de vivienda como temática en el ámbito de las ciencias sociales. Todo ello ha permitido al autor realizar esta obra donde sintetiza, con un enfoque comparativo, las principales características de los movimientos por la okupación en Europa, comparándolos al mismo tiempo con otros movimientos sociales contemporáneos y pasados, y cómo han sido influenciados por contextos sociopolíticos y urbanos específicos.

Squatters in The Capitalist City. Housing, Justice, and Urban Politics es sin duda un libro indispensable para cualquier sociólogo/a que quiera entender las principales claves explicativas de los movimientos de okupación. Este libro ofrece un análisis de estos movimientos en varios países y ciudades europeas, al combinar el propio trabajo de campo de Miguel A. Martínez y una abundante evidencia significativa recopilada de la investigación de otros. Su objetivo es revelar patrones similares que conectan transnacionalmente estas luchas locales. También persigue dar cuenta de su diversidad interna, de los límites de este tipo de política radical y de las circunstancias, procesos y características importantes que la definieron como un movimiento urbano. En este sentido y de forma general, la acción directa, la autogestión y las formas de vida comunitarias que 
han promovido estos movimientos en toda Europa han sido un foco de resistencia a la imposición del neoliberalismo en las ciudades-empresa de la actual Europa del Capital.

Los movimientos por la okupación han supuesto una relativa renovación de los movimientos urbanos, caracterizada por una reformulación de su discurso y sus objetivos en el marco de la crisis ecológica, la lucha contra la carestía de la vivienda y contra el tráfico motorizado y contaminante, así como la apuesta por nuevas formas de ocio y estilos de vida cotidiana, que plantean formas de vida que valga la pena vivir.

Ahora bien, ¿qué es okupar? En primer lugar, okupar es vivir en (o usar de otra manera) inmuebles sin el consentimiento de su propietario (Pruijt, 2004, p. 35). Podríamos añadir que se trata de un movimiento que se centra en el acceso directo a un bien urbano relativamente escaso (la vivienda y los espacios de sociabilidad) y su legítima defensa (Martínez, 2004, p. 62). El autor del libro añade que otro requisito para la okupación es que el inmueble esté previamente abandonado, por lo que la entrada en viviendas cuando los propietarios están de viaje o en segundas residencias no es okupación, sino allanamiento de morada. Por otro lado, Martínez también descarta estudiar okupaciones de la extrema derecha, de grupos delincuenciales o de grupos marginales relacionados con el tráfico y consumo de drogas.

El autor destaca que, dadas estas condiciones, la okupación aparece en contextos donde las políticas urbanas y de vivienda resultan fallidas, y lo hace desafiando a la especulación inmobiliaria y no tanto al derecho a la propiedad privada (p. 7). En otras palabras, el interés en los movimientos de okupación radica en su abordaje de las inequidades centrales del mercado inmobiliario y su gobernanza neoliberal por parte de las autoridades estatales. Los okupas son a menudo el primer frente de lucha de movimientos más amplios contra los desahucios, la violencia inmobiliaria, el incremento de los alquileres y la gentrificación. La okupación no es solo una alternativa de vida cotidiana inmediata y práctica al capitalismo, sino también una herramienta para cambiar las políticas neoliberales urbanas y de vivienda en las ciudades.

Otra de las claves del libro es la cuestión de la criminalización de la okupación en Europa, en concordancia con el ascenso de diferentes variantes del neoliberalismo en todos los gobiernos. La ideología neoliberal dominante desde finales de los años ochenta en Europa, que impone la privatización, el crecimiento económico ilimitado y la búsqueda del máximo beneficio para el mercado, es incompatible con los valores solidarios y comunitarios del movimiento okupa, que se ha visto crecientemente asediado por desalojos y persecución judicial y policial.

De todos modos, existen y conviven diversas prácticas de okupación en las ciudades europeas. Por sentido común, podríamos distinguir aquellas que se dedican a satisfacer una necesidad de vivienda, de las que se convierten en CSO donde realizar todo tipo de actividades contraculturales en un espacio público no estatal -fuera de las lógicas burocratizadas (del estado) o mercantilizadas (del sector privado) (González, 2016). Existe entonces una gran variedad de okupas y de okupaciones, aunque la mayoría tienen en común su gran esfuerzo para cuidar los lugares okupados, promover formas de vida comunales y compartir sus ideas con los vecinos. Aunque las okupaciones son poco conocidas y a menudo lo son solo por los desalojos, este libro en un impresionante testimonio de miles de actividades culturales, charlas, talleres y, en general, del fomento de la sociabilidad alternativa que han protagonizado varias generaciones de okupas y simpatizantes de este movimiento. Por desgracia, cuando son los más pobres y necesitados los que utilizan la okupación de inmuebles, suelen ser incluso más reprimidos, a pesar de que en la Unión Europea existen 11 millones de casas vacías y más de 4 millones de personas que carecen de hogar (p.9). 
A pesar esta inmensa variedad y pluralidad, Squatters in The Capitalist City consigue identificar los rasgos característicos de los movimientos por la okupación en Europa en los últimos 40 años. En primer lugar, los okupas suelen ser ciudadanía activa que consigue soluciones asequibles y autónomas a complejos problemas de la vida en las ciudades, como el acceso a la vivienda o la posibilidad de crear alternativas culturales desde la base. En segundo lugar, las okupaciones son instituciones culturales anómalas, al ser espacios comunales urbanos, ni públicos, ni privados. En tercer lugar, cabe destacar su contribución a la justicia social y la equidad, al integrar a sectores sociales potencialmente excluidos de la vida cultural, comunitaria y política (Calle, 2004). En cuarto lugar, las okupaciones actúan a menudo como desarrolladores urbanos culturales y protectores de espacios, que, a pesar de su interés, se están deteriorando por su abandono o van a desaparecer fruto de la voracidad de la especulación inmobiliaria. Finalmente, en todos los países -a pesar del predominio de la lógica neoliberal- existen diversas regulaciones legales o procesos de reconocimiento de las okupaciones y acceso a la propiedad de inmuebles abandonados, como el derecho de usucapión en España.

Por lo que se refiere a la estructura del libro, éste cuenta con 6 capítulos, de los cuales procederemos a hacer un comentario sintético, destacando sus principales aportaciones, y recomendando encarecidamente su lectura.

El primer capítulo "Squatting as an Urban Movement" (pp. 13-62), justifica por qué el análisis de los movimientos urbanos necesita unir los hallazgos de la economía política y de la política contenciosa. Define los movimientos urbanos de acuerdo con los contextos influyentes del capitalismo y la democracia, y distingue los movimientos de las prácticas activistas de corta duración y no coordinadas. Una erudita revisión de la literatura académica (Castells, Villasante, Harvey, Pickvance, Tilly, Tarrow, Mayer, Foucault o Jessop, entre otros) permite al autor la identificación de las principales condiciones estructural-sistémicas de los movimientos urbanos y su producción social contenciosa del espacio. Miguel A. Martínez aplica magistralmente estos supuestos teóricos y elecciones conceptuales para explicar el aumento, el desarrollo y las consecuencias de las okupaciones en Europa, como expresión clave de los movimientos urbanos.

El segundo capítulo, titulado "Autonomy from Capitalism" (pp. 63-96), examina el papel que jugó la noción de "autonomía" en la política urbana de los movimientos de okupación en Europa, pero actualizándola desde las nuevas perspectivas del derecho a la ciudad y los comunes urbanos (según Lefevbre, Mitchell, Marcuse, Fraeser, entre otras) para comprender cómo los movimientos sociales del ámbito de la autonomía -en la frontera entre el anarquismo y la izquierda libertaria-radical (Della Porta y Rucht, 1995)- proporcionaron una referencia de identidad al movimiento okupa. El autor sugiere centrarse en las dimensiones de autonomía colectiva (Katsiaficas), feminismo (Balestrini, Moroni, Federici, Gil) y anticapitalismo (Geronimo, Flesher) para comprender la formación de la identidad, los repertorios de protesta y el magma de redes políticas en las que se integraron los movimientos okupas europeos. Breves análisis históricos de Italia, Alemania y España ilustran este enfoque.

A continuación, el tercer capítulo - "Socio-Spatial Structures" (pp. 97-138)- investiga cómo emergen y se desarrollan las okupaciones, es decir, los condicionantes estructurales de la acción estratégica del movimiento. Estas estructuras pueden ser de naturaleza local, pero a menudo se forman a escala nacional y siguen las tendencias internacionales, en paralelo con las redes transnacionales de okupas. Los activistas interpretan estas estructuras e intentan descubrir grietas que permitan hacer prosperar sus prácticas disruptivas, mientras que las autoridades ejercen su influencia en estas estructuras para suprimir, regular o evitar la extensión de las okupaciones. La discusión sobre el urbanismo neoliberal, la gentrificación y la regulación de la okupación, se ilustra mediante un análisis específico de la trayectoria del movimiento okupa en Amsterdam. 
"Types of Squatting" es el título del cuarto capítulo (pp. 141-190), que como su nombre indica, da cuenta de forma crítica de los diversos intentos de categorizar el mundo de las okupaciones (de acuerdo a Aguilera, González, Polanska, Pruijt e incluso el propio Martínez), proponiendo una tipología comprensiva alternativa (p. 152) con siete categorías distintas: 1/provisión de vivienda; 2 / resistencia a los desahucios; 3 /defensa de los bienes comunes; $4 /$ beneficios medio-ambientales; $5 /$ cultura alternativa; $6 /$ infraestructura política para la agregación social y el empoderamiento democrático; y $7 /$ derecho a la ciudad (espacios seguros para mujeres, personas LGBTQI, minorías étnicas e inmigrantes-refugiados). En esta nueva clasificación distingue, como decíamos anteriormente, entre okupaciones de vivienda y CSO, pero también entre okupación táctica y estratégica, con el objetivo de clarificar la dimensión política de las prácticas y movimientos de okupación. Dos estudios de caso sobre la articulación entre migración y okupación en Madrid, y sobre las divisiones entre diferentes tipos de okupaciones en su relación con las autoridades locales y las políticas urbanas en París, muestran estas nuevas claves.

El capítulo 5 se titula "Anomalous Institutions" (pp. 191-233) y sintetiza una de las aportaciones más originales a la sociología urbana de Miguel A. Martínez, la cuestión de los procesos de legalización y de los outcomes de la okupación. Los trabajos de Hans Pruijt (2003) sobre la institucionalización de los movimientos okupas en Amsterdam y Nueva York sirvieron de inspiración a Martínez para profundizar tanto a nivel conceptual, como empírico. Primero, analiza las "negociaciones estratégicas" entre los okupas y sus oponentes, distinguiendo entre negociaciones de alto nivel, transaccionales, de supervivencia y forzadas. A continuación, analiza en qué medida la legalización se considera una condición para una larga duración las okupaciones, dadas las escasas oportunidades de convertir las okupaciones en formas legales de tenencia. Distingue tres tipos de procesos de "institucionalización": la integración en las instituciones del estado, la promoción de nuevas instituciones y finalmente, la creación de "instituciones anómalas" como una forma de entender la resistencia de los okupas a la asimilación del Estado. Se incluye otro estudio de caso de Madrid para ilustrar y concretar todo este desarrollo teórico, especialmente original.

Finalmente, el sexto capítulo "Criminalisation and Counter-Hegemony" (pp. 234-270), pone el broche final a esta extraordinaria obra con un análisis de los patrones comunes de los procesos de criminalización de la okupación en Europa. Los medios de comunicación y las élites utilizan dos estrategias retóricas para estigmatizar las prácticas de okupación, una de "estigmatización total" (que acusa a los okupas de alterar el orden social y comportarse de forma inmoral) y otra de "estigmatización parcial" (que selecciona a la porción de okupas más funcional a la reproducción de la ciudad capitalista: artistas y creativos de clase media capaces de ser pioneros en los procesos de gentrificación). La intención de esas estrategias es dividir y enfrentar a los propios okupas entre sí, además de socavar la legitimidad social de sus prácticas. Sin embargo, los okupas responden con potentes marcos de legitimación de las mismas, tanto subversivos como reversivos, de manera que el conflicto entre las narrativas hegemónicas y contrahegemónicas muestra con más claridad si cabe, las cuestiones estructurales con respecto al sistema capitalista y la lucha de clases que están implícitas en la okupación de inmuebles abandonados. 


\section{REFERENCIAS BIBLIOGRÁFICAS}

Calle, Á. (2004). Okupaciones: un movimiento contra las desigualdades materiales y expresivas. En F. Tezanos (ed.), Tendencias en desigualdad y exclusión, Madrid: Sistema, pp. 270-305.

Della Porta, D. y Rucht, D. (1995). Left-Libertarian Movements in Context: A Comparison of Italy and West Germany 1965-1990. En J. Craig Jenkins y B. Klandermans (eds.) The Politics of Social Protest: Comparative Perspectives on States and Social Movements (pp. 229-273). London: UCL Press.

González, R. (2016). Movimientos Sociales y Vivienda en España. Boletín Científico Sapiens Research, 6(1), 31-35.

Martínez, M.A. (2004). Del urbanismo a la autogestión: una historia posible del movimiento de okupación en España. En R. Adell y M.A. Martínez (coords.), ¿Dónde están las llaves? El movimiento okupa: prácticas y contextos sociales (pp. 61-88). Madrid: Los -libros de la Catarata.

Pruijt, H. (2003). Is the Institutionalization of Urban Movements Inevitable? A Comparison of the Oportunities for Sustained Squatting in New Cork City and Amsterdam. International Journal of Urban and Regional Research, 27(1), 133-157.

http://dx.doi.org/10.1111/1468-2427.00436

Pruijt, H. (2004). Okupar en Europa. En R. Adell y M. A. Martínez (coords.), ¿Dónde están las llaves? El movimiento okupa: prácticas y contextos sociales (pp. 35-60). Madrid: Los libros de la Catarata. 
